\title{
Design and dynamic characterization of a gyroscopic system for aerobatic UAV helicopters
}

\author{
Antonio Affanni* \\ University of Udine, Dept. of Electrical, Management and Mechanical Engineering, Via delle Scienze 208, 33100 Udine, Italy
}

\section{A R T I C L E I N F O}

\section{Article history:}

Received 20 March 2012

Received in revised form 19 June 2012

Accepted 11 September 2012

Available online 13 October 2012

\section{Keywords:}

Attitude control

Servosystems

Electromechanical sensors

\begin{abstract}
A B S T R A C T
This paper describes the design, development and dynamic characterization of a high performance MEMS-based gyroscopic control system for the yaw channel of Unmanned Aerial Vehicles (UAVs) Radio Controlled (RC) helicopters for aerobatic maneuvers. A new asymmetrical controller has been developed that compensates the torque of the main rotor thus providing equal dynamic response in clockwise and anticlockwise pirouettes. The "in flight" dynamic characterization showed that the proposed system can be up to five times faster than the state of the art for commercial gyros at higher yaw rates; the regime yaw rate characterization demonstrated a high and constant pirouette speed. Aerobatic tests demonstrated high accuracy entry into the maneuvers.
\end{abstract}

(c) 2012 Elsevier Ltd. All rights reserved.

\section{Introduction}

The high maneuverability of miniature helicopters can be useful for aerial surveillance in complex confined environments such as search and rescue scenarios from earthquakes or, in general, in many applications in which full size vehicles cannot access the target sites. This led to an increasing interest in these vehicles in the Scientific Community that, in the last decade, focused on mathematical models [1-4], control solutions with multisensors platforms [5-10], mounting commercial gyroscopic systems on board [11-15], or studying the fault detection of sensors and actuators mounted on board [16]. At present the main employment of such helicopters is in aerobatic international competitions, where aggressive maneuvering is imposed by the pilots and the system must respond rapidly and precisely. Maneuvers like "Tic Toc", "Funnel", "Piroloop", "Piroflip", and "Chaos" [17] are performed in few tens of milliseconds and the full attitude of the vehicle is very complex to be manually controlled; if the yaw axis is automatically regulated, the maneuverability of these vehicles is strongly increased. In order to aid the pilot in precise

\footnotetext{
* Tel.: +39 0432558034.

E-mail address: antonio.affanni@uniud.it
}

maneuvering, it is necessary to design an electronic feedback control system with optimal bandwidth; in particular the control of the yaw channel is a challenging task because high precision and velocity are requested to the system which controls the tail position and acts on the tail rotor blades. This paper focuses on the design and characterization of a gyroscopic control system whose performances are optimized for aerobatic competitions; the yaw channel has been modelled and a Proportional-Integrative controller has been designed, as in [18,19], but the very aggressive environment of operation brought to the design of a very fast asymmetric switched control.

In [20] we showed the design choices and the simulations of a MEMS-based gyroscopic system providing only preliminary experimental data obtained on a turntable. With the present work we provide the dynamic characterization of the yaw rate behaviour obtained from measurements "in flight". Even if measurements performed on a turntable could provide higher accuracy, however the turntable friction can introduce artifacts not corresponding to the real helicopter behaviour during flight. For this reason we preferred to test the system "in flight" and compare its performances with the performances of the best performing available commercial gyroscopes (our benchmark). Measurements showed that the designed system 
allows entry into maneuvers up to five times faster than the benchmark, guaranteeing higher precision of tail control in aerobatic flight.

The paper is organized as follows. Section 2 shows the description of the helicopter and an overview of the performances requested to the gyroscopic system. Sections 3 and 4 recall the results obtained in [20] and, respectively, show a derivation of the helicopter yaw channel dynamic model with its characterization and the simulations results of the proposed control loop compared to the benchmark. Finally, Section 5 provides the flight test results obtained during aerobatic maneuvers, in terms of regime yaw rate and step dynamic response. The repeatability of the step response is also evaluated by means of the acquisition of several aerobatic pirouettes.

\section{System description}

The gyroscopic system has been designed on a commercial Radio Controlled helicopter model powered by a 0.5 cubic inches alcohol-engine, with an overall mass of $4 \mathrm{~kg}$; a view of the helicopter used for the design is shown in Fig. 1. The fuselage length is $1150 \mathrm{~mm}$, the diameter of the two blades main rotor (tail rotor) is $1370 \mathrm{~mm}$ (192 mm, respectively). The blade movement is actuated by commercial servomotors which need Pulse Position Modulated (PPM) signals with standard durations of $1520 \mu$ s or $760 \mu \mathrm{s}$. The main rotor speed is variable up to $2200 \mathrm{RPM}$ and the engine speed is up to $21,000 \mathrm{RPM}$.

The present gyroscopic system has been described in [20]. In this and next sections we summarize the design steps and choices that brought to the experimental results presented in Section 5.

As shown in Fig. 2a, the system is composed by a MEMS gyroscopic sensor, a filter and a digital signal processor (DSP). The MEMS gyroscope gives information on the tail yaw rate and the DSP provides correct output to the servomotor which acts on the tail rotor blades. A hardware filter with steep frequency response has been inserted between the sensor and the DSP to reduce noise due to mechanical vibrations generated by the main rotor and engine. The DSP receives three inputs and must provide an adequate output for the servomotor which moves the tail rotor blades. Two inputs are standard PPM modulated digital signals, coming from the RC receiver and corresponding to two signals set by the pilot: Stick and Gain. Stick signal

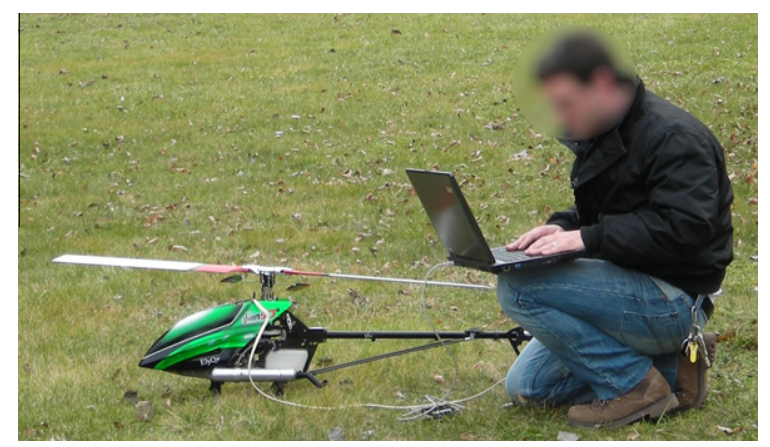

Fig. 1. RC helicopter used for the design of gyroscopic system. is the setpoint and represents the desired yaw rate of the helicopter. Gain signal is used to set the dynamic response of the system, i. e. how aggressive the control must be in aerobatic maneuvers.

Maximum required controlled yaw rate and transient response time in hard maneuvers entrance are known as the most strict requirements for the gyroscopic system. The first step in the design has been the choice of a low cost MEMS-based gyroscopic sensor capable of reaching high yaw rates (up to $\pm 500 \% / \mathrm{s}$ ) with a good accuracy. Unfortunately all available low cost MEMS gyroscopes have a maximum input range of $\pm 300 \% \mathrm{~s}$, and this value, too low, does not meet the needs of the pilots.

The choice of the sensor fell on the ADXRS620 by Analog Devices, which provides an analog voltage output centered in $2.5 \mathrm{~V}$ and spanning $\pm 1.8 \mathrm{~V}$ at a full scale yaw rate of $\pm 300^{\circ} /$ $\mathrm{s}$ with an accuracy of $0.1 \%$ of full scale. In order to increase the pirouette speed, as requested by RC pilots, it is possible to extend the input range by reducing the gain of the MEMS conditioning circuitry. The declared maximum yaw rate, in fact, is not limited by the maximum angular rate which can be measured by the proof mass, but by the saturation of the on board conditioning circuitry. Through the proper selection of an external resistor connected to the gyroscope pins RATEOUT and SUMJ [21], it is possible to lower the gain of the conditioning circuitry and to increase the full scale angular rate on the expense of accuracy as demonstrated in Section 5.1. In the designed system the full scale yaw rate has been increased up to a nominal value of $\pm 600^{\circ} / \mathrm{s}$, with a further overrange up to $\pm 660 \%$ s. The sensor output has been connected to a double pole Sallen-Key low pass filter; the filter has been designed to obtain two coincident poles at $50 \mathrm{~Hz}$ yielding to an overall $-3 \mathrm{~dB}$ cutoff frequency of $30 \mathrm{~Hz}$. The aim of the filter is to reduce the effect of mechanical vibrations, with this design the rotor and engine vibrations are attenuated by $4 \mathrm{~dB}$ and $34 \mathrm{~dB}$, respectively. The active filter output voltage is then acquired by an Analogto-Digital Converter (ADC) on board on DSP. Fig. 2a shows the block diagram of the described system and Fig. $2 b$ shows the realized $22 \mathrm{~mm} \times 22 \mathrm{~mm}$ prototype board.

The chosen DSP is a Microchip DSPIC 30F3013, characterized by a 16 bit core and 12 bit ADC. Stick and Gain signals are captured by the DSP with 11 bits resolution, while the measured yaw rate is acquired by the ADC with 12 bits resolution. The DSP output is a PPM modulated signal for standard servomotors with programmable duration of $760 \mu$ s or $1520 \mu$ s.

\section{Helicopter characterization and system design}

The modelling and characterization of the helicopter dynamic behaviour is mandatory when an optimized response in terms of speed and stability is required. The yaw channel, which is composed by the servomotor and the tail blades, can be described as a transfer function between the normalized servomotor position $\delta_{M} \in[-1,1]$ and the yaw angular rate $\omega_{z}$. Then, the sensor-based feedback system senses $\omega_{z}$ and provides its filtered, quantized and re-normalized value $\delta_{\omega} \in[-1,1]$ to the controller which, in turn, feeds the servomotor position $\delta_{M}$ to the yaw channel. 


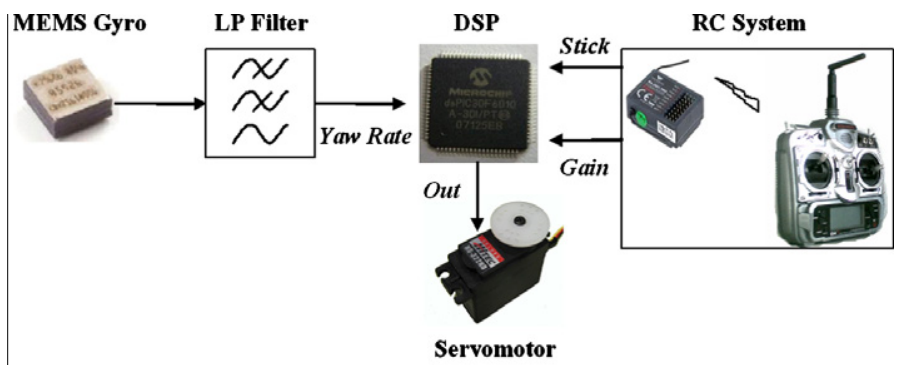

(a)

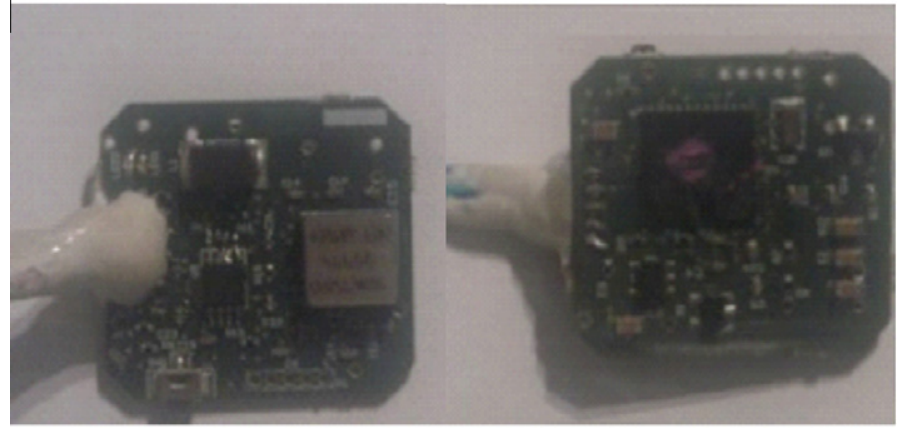

(b)

Fig. 2. (a) Block diagram of the described system and (b) prototype of the gyroscopic system. Dimensions are $22 \mathrm{~mm} \times 22 \mathrm{~mm}$.

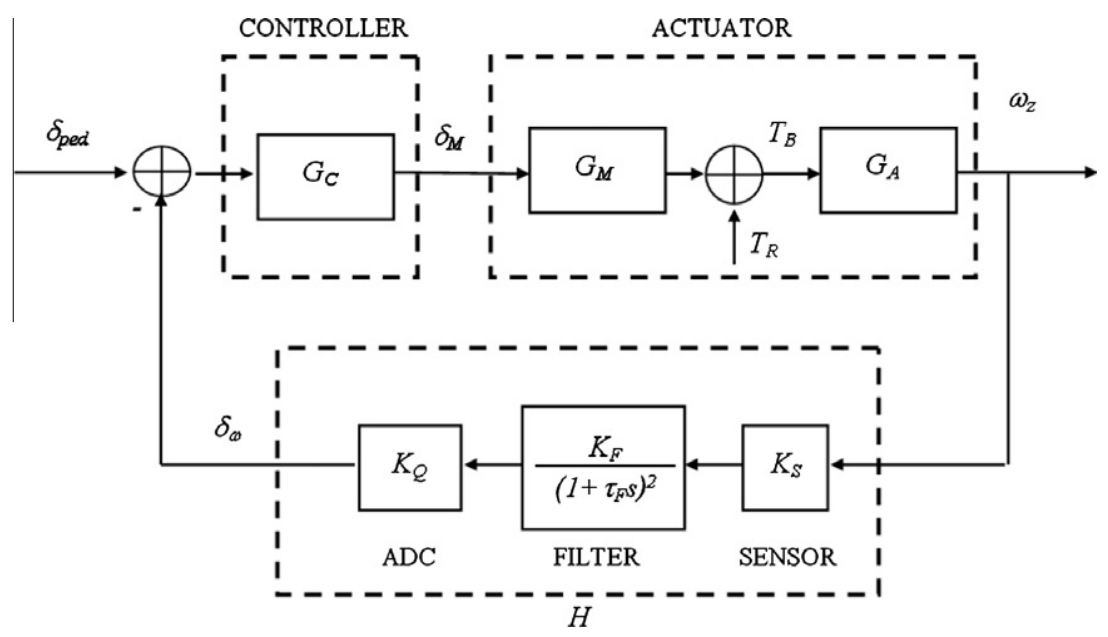

Fig. 3. Block diagram of the yaw channel dynamic model.

In the yaw channel, the servomotor of the tail blades can be modelled as a first order transfer function

$G_{M}(s)=\frac{K_{M}}{\tau_{M} s+1}$

with a time constant $\tau_{M}$ typically in the range $[0.1,0.3] \mathrm{s}$. The relationship between tail blades torque $T_{B}$ and yaw rate is

$G_{A}(s) \equiv \frac{\omega_{z}(s)}{T_{B}(s)}=\frac{1}{I s+D}$ where $I$ is the moment of inertia around the main rotor axis and $D$ the viscous friction acting on the rotating tail. Notice that the state-of-the-art gyroscopic systems are based on the hypothesis that the behaviour of the yaw channel is symmetrical with respect to the yaw direction (i. e. $T_{R}=0$ in Fig. 3). Helicopters, however, are highly asymmetric systems because of the presence of main rotor torque $T_{R}$, which acts always in the same direction, opposite to the main rotor blades angular rotation. This means that the response for clockwise or anticlockwise yaw rates is different and, if the torque $T_{R}$ significantly contributes to 
$T_{B}$, the system bandwidth is dramatically reduced. By taking into account the torque $T_{R}$, the transfer function of the yaw channel becomes

$T_{B}(s)=G_{M}(s) \delta_{M}(s)+T_{R}(s)$

The feedback block converts the input quantity $\omega_{z}$ into a normalized quantity $\delta_{\omega}$ that is the numerical value of the yaw rate quantized by the ADC on board on DSP. This transfer function involves the sensor with expanded full scale yaw rate at $\pm 600 \% \mathrm{~s}$ followed by the hardware Sallen-Key filter with double pole. The transfer function is

$H(s) \equiv \frac{\delta_{\omega}(s)}{\omega_{z}(s)}=\frac{K_{Q} K_{F} K_{S}}{\left(\tau_{F} s+1\right)^{2}}$

where $K_{S}$ is the sensitivity of the sensor, $K_{F}$ is the gain of the active filter, $\tau_{F}$ is the time constant of the Sallen-Key filter and $K_{Q}$ is the analog to digital converter gain which converts the filter output voltage into the normalized quantity $\delta_{\omega}$.

The controller typology implemented on the DSP is a modified Proportional-Integrative (PI) solution, with asymmetrical gains to overcome the asymmetric behaviour of the helicopter as seen above.

$G_{C}(s)=\frac{K_{P} s+K_{I}}{s}$

Finally an anti-windup algorithm named "integrator clamping" [22,23], has been implemented yielding to an important improvement of the high yaw rate dynamic response with respect to our benchmark as discussed in next sections. In digital controllers in fact the anti-windup algorithm assumes a high relevance; since all actuators have limited input and output ranges, the controller must provide an adequate output for the actuator even when the feedback loop does not reach the setpoint. On the other hand, the integral component can theoretically diverge to infinity (or in practice numerically overflow) if the actuator is working at its limits (i.e. in saturation zones); even when overflow does not occur, a too high value of the integral component slows the controlled system. Anti-windup algorithm acts blocking the integral component and thus preventing the excessive integration when actuator is saturated. The most common algorithm is "bounding integration", i.e. setting fixed upper and lower bounds for the integral; this algorithm, though very simple, has many drawbacks since the integral discharge time depends on the setpoint and is very susceptible to the system parameters variability. The "integrator clamping" algorithm instead, recognizes why a saturation of the output has been reached: if the setpoint signal and output have the same sign it means that further increase of the integral is not useful so the integral is locked at its "last good" value until the output exits from saturation. Since the integral component is locked to a reasonable value instead of being bounded to a fixed limit, the "integrator clamping" algorithm provides a faster dynamic response than "bounded integrator".

All the dynamic parameters of helicopter have been measured, with the exception of $\tau_{M}$ in (1) (fixed to $0.16 \mathrm{~s}$ from servomotor datasheet) and $I$ in (2) (approximated to a rotating bar with $0.01 \mathrm{~kg} \mathrm{~m}^{2}$ ). The torque $T_{B}$ has been derived from the measurement of the force (through a dynamometer) produced by the tail blades when $\delta_{M}= \pm 1$ is applied. It results $-1 \pm 0.05 \mathrm{~N} \mathrm{~m}$ clockwise and $0.5 \pm 0.05 \mathrm{~N} \mathrm{~m}$ anticlockwise. Thus, from (3) $K_{M}=0.75 \pm 0.05 \mathrm{~N} \mathrm{~m}$ and $T_{R}=0.25 \pm 0.05 \mathrm{~N} \mathrm{~m}$. The regime open-loop yaw rate $\omega_{z}$ has been measured posing $\delta_{M}= \pm 1$ and fastening the helicopter on a low friction turntable; a video with a camera has been acquired at a frame rate of 25 fps during a 10 turns pirouette in clockwise and anticlockwise directions and the estimation of $\omega_{z}$ has been derived from the number of frames elapsed during the 10 turns pirouette. Neglecting the frame rate accuracy, the accuracy of the measurement of $\omega_{z}$ depends only on the accuracy of the angle readout from the first and last frames. With this setup, when $\delta_{M}=-1$ it is obtained $\omega_{z}=-27 \pm 0.5 \mathrm{rad} / \mathrm{s}$ in clockwise direction and when $\delta_{M}=1$ it is obtained $\omega_{z}=13 \pm 0.1 \mathrm{rad} / \mathrm{s}$ in anticlockwise direction; then, from (2), $D=0.037 \pm 0.002 \mathrm{~N} \mathrm{~m} \mathrm{~s} / \mathrm{rad}$ is found.

The model parameters of the feedback transfer function (4) have been derived from sensor and ADC datasheets, providing $K_{S}=0.172 \pm 2 \times 10^{-4} \mathrm{~V}$ s/rad, $K_{F}=1.4 \pm 3 \times 10^{-2}$, $\tau_{F}=3.2 \mathrm{~ms}$ and $K_{Q}=0.4 \pm 4 \times 10^{-4} 1 / \mathrm{V}$.

The controller parameters have been finally designed taking into account the model parameters shown above. The ratio $K_{I} / K_{P}$ in (5) has been set in the order of $10 \mathrm{rad} / \mathrm{s}$, slightly higher than the quantity $D / I$ in (2); with this choice the closed-loop transfer function will behave approximately as a second order system with a damping factor in the order of 0.8, guaranteeing a high speed step response with a negligible overshoot. The $K_{P}$ gain varies accordingly to two control signals, namely the RC pilot desired bandwidth and the requested pirouette direction (i. e. clockwise or anticlockwise). The pilot can set the control bandwidth by sending the radio signal gain to the system and can obtain a very fast response (expert pilot and hard aerobatic maneuvers) or a slower response (practicing pilot) of the system. Defining $K_{\text {Pclock }}$ and $K_{\text {Panticlock }}$ the proportional gain in clockwise and anticlockwise direction respectively, the quantity $K_{\text {Pclock }}$ is derived from the Gain signal and, choosing $K_{\text {Panticlock }} / K_{\text {Pclock }} \simeq 2$ as a constant ratio, has shown the best response in simulations and experimental results.

The complete closed-loop transfer function which converts the pilot setpoint $\delta_{\text {ped }} \in[-1,1]$ into the angular rate $\omega_{z}$ and considers also the main rotor torque is thus

$$
\begin{aligned}
\omega_{z}(s)= & \frac{G_{C}(s) \cdot G_{M}(s) \cdot G_{A}(s)}{1+G_{C}(s) \cdot G_{M}(s) \cdot G_{A}(s) \cdot H(s)} \cdot \delta_{\text {ped }}(s) \\
& +\frac{G_{A}(s)}{1+G_{C}(s) \cdot G_{M}(s) \cdot G_{A}(s) \cdot H(s)} \cdot T_{R}(s)
\end{aligned}
$$

\section{Simulations results}

The presented gyroscopic system differs from available commercial ones (our benchmark) mainly for two design choices: an asymmetrical control and an improved antiwindup algorithm. In [20] not only time-domain, but also frequency-domain simulations were presented, which in the present paper are omitted. In this section we just report the simulations which can be compared with the performed measurements, with the same setpoint patterns and amplitudes. The simulations of step responses of our 


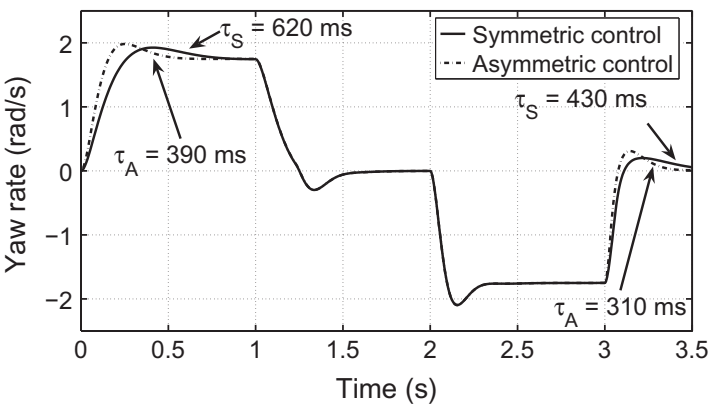

Fig. 4. Simulation comparison between symmetric and asymmetric control as response of multiple steps of amplitude $\pm 1.75 \mathrm{rad} / \mathrm{s}$; arrows represent the setpoint $\pm 5 \%$.

system are shown with comparison with the benchmark of commercial gyroscopic systems pointing out the improvements due to the different design choices. The system response to a small amplitude step will be shown as a first interesting result, which can be useful to show the effective improvement of the asymmetric control when the output is not saturated and the anti-windup algorithm is not working. Fig. 4 shows the response to a setpoint yaw rate pattern composed by consequent steps of amplitude $\pm 1.75 \mathrm{rad} / \mathrm{s}$, each step has a duration of $1 \mathrm{~s}$.

The step response for an anticlockwise yaw rate (in Fig. 4 and next ones with the convention of positive yaw rate) with symmetric control is much slower than with the asymmetric control, since in the designed asymmetric control $K_{\text {Panticlock }} / K_{\text {Pclock }} \approx 2$ while in symmetric control $K_{P} \equiv K_{\text {Pclock }}$ and anticlockwise steps are controlled with a proportional term that is one half with respect to asymmetric control; defining $\tau_{A}$ and $\tau_{S}$ the times necessary to reach the $\pm 5 \%$ setpoint for asymmetric control and for symmetric control respectively, it is possible to see that $\tau_{A}$ is considerably shorter than $\tau_{s}$. Also in case of a stopping step, it is possible to observe a significant improvement of the system due to the choice of an asymmetrical control. Moreover, with the choice of $K_{\text {Panticlock }} / K_{\text {Pclock }} \approx 2$, the response is almost symmetric.

A second set of simulations has been performed with a yaw rate setpoint pattern of steps with high amplitude regime value, precisely $\pm 8 \mathrm{rad} / \mathrm{s}$, each step has a duration of $1 \mathrm{~s}$. Such high amplitude allows comparing the behaviour of the chosen anti-windup "integrator clamping" with the state-of-the-art gyroscopic controllers.

Fig. 5 shows the step response when the anti-windup algorithm blocks the integration of the controller. Defining $\tau_{P}$ and $\tau_{B}$ the times necessary to reach the setpoint $\pm 5 \%$ for proposed system and for benchmark algorithm "bounded integration" respectively, it is possible to see that $\tau_{P}$ is much lower than $\tau_{B}$ and the proposed system is faster than the state of the art also in case of output saturated. For completeness, all simulations have been repeated taking into account the non-linearity of MEMS sensor, accordingly to the sensor characterization presented in Section 5.1; results showed that sensor non-linearity mainly influences the regime values of yaw rate. As shown in the next section, the non-linearity is less than $1 \%$ of sensor full scale (i.e. $0.1 \mathrm{rad} / \mathrm{s}$ ), so that the regime yaw rates in Figs. 4 and

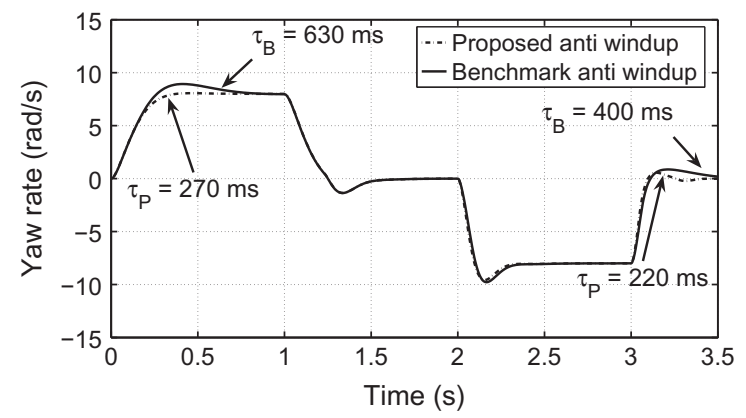

Fig. 5. Simulation comparison between the proposed anti-windup "integrator clamping" and the benchmark solution "bounding integration" as response of multiple steps of amplitude $\pm 8 \mathrm{rad} / \mathrm{s}$; arrows represent the setpoint $\pm 5 \%$.

5 are modified by the same quantity. On the contrary, settling times shown in Figs. 4 and 5 are not significantly affected by sensor accuracy.

\section{Experimental characterization}

\subsection{MEMS sensor and filter characterization}

The MEMS gyroscopic sensor has been characterized in terms of linearity performances both in the case of the sensor with original input range, $\pm 300^{\circ} / \mathrm{s}$, and the sensor with extended input range, $\pm 600^{\circ} / \mathrm{s}$. The measurements have been performed mounting the gyroscopic sensor and a portable digital multimeter on a motorized turntable capable of a maximum angular speed of $\pm 550 \%$ s. The angular speed has been derived acquiring the rotating system with a camera at a frame rate of $25 \mathrm{fps}$; since the relative uncertainty of the angular rate is $\frac{u(\omega)}{\omega}=\frac{1}{\sqrt{3} N_{F}}$ (being $N_{F}$ the number of acquired frames and having considered the camera frame discontinuity uncertainty distributed in $[-1,1]$ with a uniform Probability Density Function), in each measurement the turntable has been filmed for $45 \mathrm{~s}$ in order to obtain $0.05 \%$ accuracy in the angular speed measurement.

Fig. 6 shows the effective increase of sensor input range, the sensitivity in fact has been reduced from $6 \mathrm{mV} / \%$ s to $3 \mathrm{mV} / \% / \mathrm{s}$; Fig. 6 shows also that the linearity, evaluated with Least Squares regression, has slightly worsened to $\pm 1 \%$ FS. Finally, Fig. 6 shows that the maximum allowed turntable speed $( \pm 550 \% \mathrm{~s})$ is quite far from sensor saturation region, thus allowing to infer that the maximum input range has been extended up to the target $\pm 600^{\circ} / \mathrm{s}$.

The double pole Sallen-Key filter has been characterized using as input signal a sine wave of variable frequency and simultaneously measuring the true RMS value of filter input and output. The characterization has been performed from $1 \mathrm{~Hz}$ to $1 \mathrm{kHz}$ with logarithmic steps and 6 points/ dec. Fig. 7 shows the measured filter transfer function, the uncertainty on transfer function data is less than $0.1 \mathrm{~dB}$.

\subsection{System characterization}

Two measurement methodologies were employed to characterize the proposed system. The characterization was not performed in laboratory on a turntable but "in 

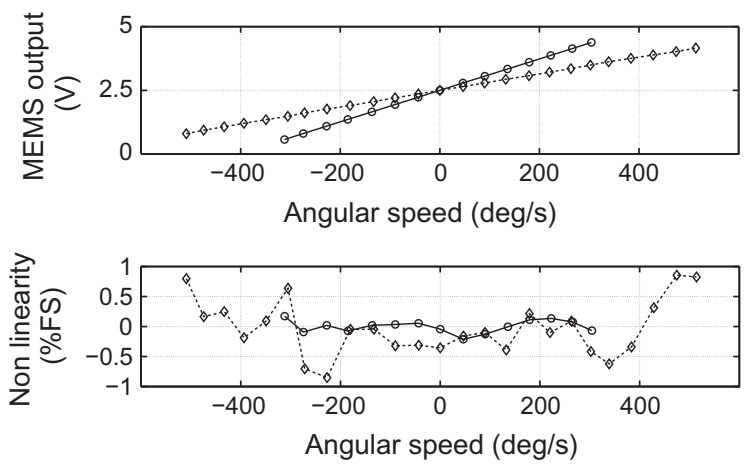

Fig. 6. MEMS sensor characterization. Linearity is evaluated with the Least Squares regression and it is presented as percentage of sensor full scale (FS). Performances of the sensor without input range extension ( $\bigcirc$ ) and with input range extension $(\diamond)$.

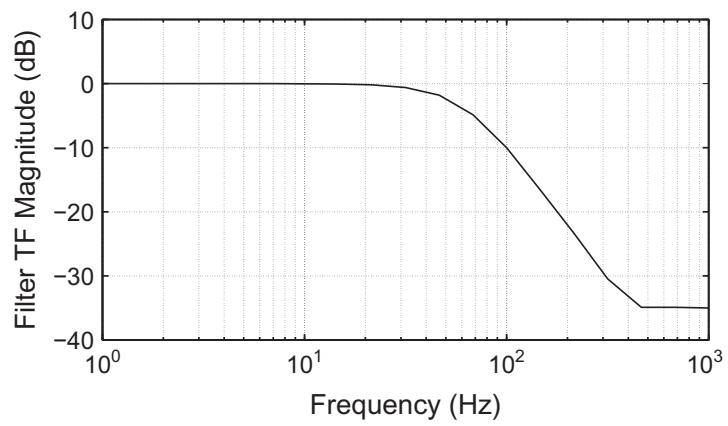

Fig. 7. Measured Sallen-Key filter transfer function (TF) magnitude.

flight", since this choice allows estimating system performances in real, much more critical conditions. Of course, a worsening of the measurement accuracy was expected with respect to the characterization on a turntable in the laboratory, because of the difficulty in performing the requested maneuvers and also the presence of modifying in- puts (e. g. not-constant wind speed and other environment conditions).

A first set of measurements pointed out an effective increase of the sensor input range, from the declared range $\pm 300^{\circ} / \mathrm{s}$ to, approximately, $\pm 600^{\circ} / \mathrm{s}$. In this test, the helicopter pirouettes were acquired at a frame rate of $25 \mathrm{fps}$ by a CCD camera with the pirouette speed ("piro rate") set at $\pm 100 \%$ on the configuration of the transmitter. At this rate, the theoretical expected value of yaw rate was $\pm 600 \% \mathrm{~s}$, approximately confirmed by the results reported in Fig. 8, where the frame sequence of the clockwise pirouette is shown over an angle range of $180^{\circ}$. Notice that the $180^{\circ}$ angle was swept in a time interval corresponding to eight frames $(0.32 \mathrm{~s})$, so that we can argue that the yaw rate in clockwise direction was slightly better than $562 \%$ s. Since the angle resolution due to the camera frame discontinuity is $(180 / 8)^{\circ}=22.5^{\circ}$, then the uncertainty on the pirouette speed estimation is $\frac{22.5^{\circ}}{0.32 \sqrt{3}} / \mathrm{s}=40^{\circ} / \mathrm{s}$, having considered the camera frame discontinuity uncertainty distributed in $[-1,1]$ with a uniform Probability Density Function. Actually, by considering the helicopter position in each frame, it is possible to state that the measurement result is slightly underestimated.

Similarly, Fig. 9 shows the good symmetry of yaw rate speed in anticlockwise direction.

A second measurement methodology was adopted to characterize the dynamic performances of the system in terms of response to different amplitude steps. Experimental data were acquired by mounting a logging circuit on the helicopter which communicated with a SD memory card and, simultaneously, stored the setpoint values $\delta_{\text {ped }}$, the servomotor output and the yaw rate $\omega_{z}$ measured by a reference gyroscopic sensor. Data were acquired synchronously with the servomotor output, at $333 \mathrm{~Hz}$ of sample rate, so that the uncertainty on the settling data presented in the following was of the order of $3 \mathrm{~ms}$.

Experiments were performed similarly to the simulations, and were repeated once with the proposed gyroscopic system and once with the benchmark. For obtaining a comparison as accurate as possible, the tests on state-of-the-art and proposed gyroscopes were per-

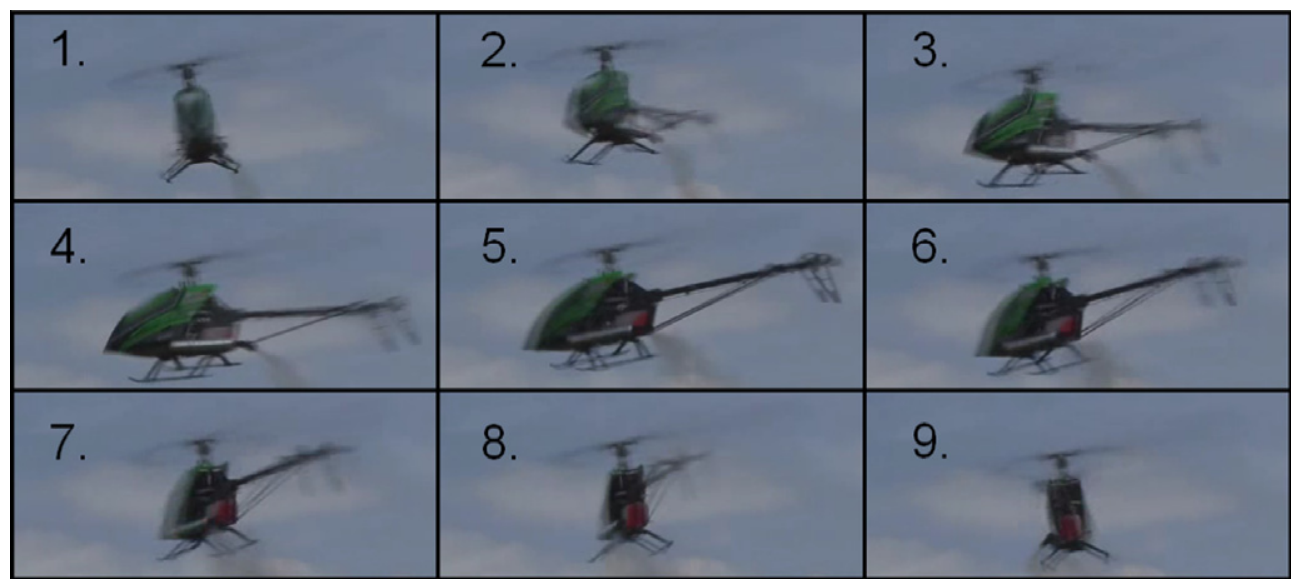

Fig. 8. Frame sequence of clockwise pirouette performed in $0.32 \mathrm{~s}$ : the angle is approximately $180^{\circ}$. 


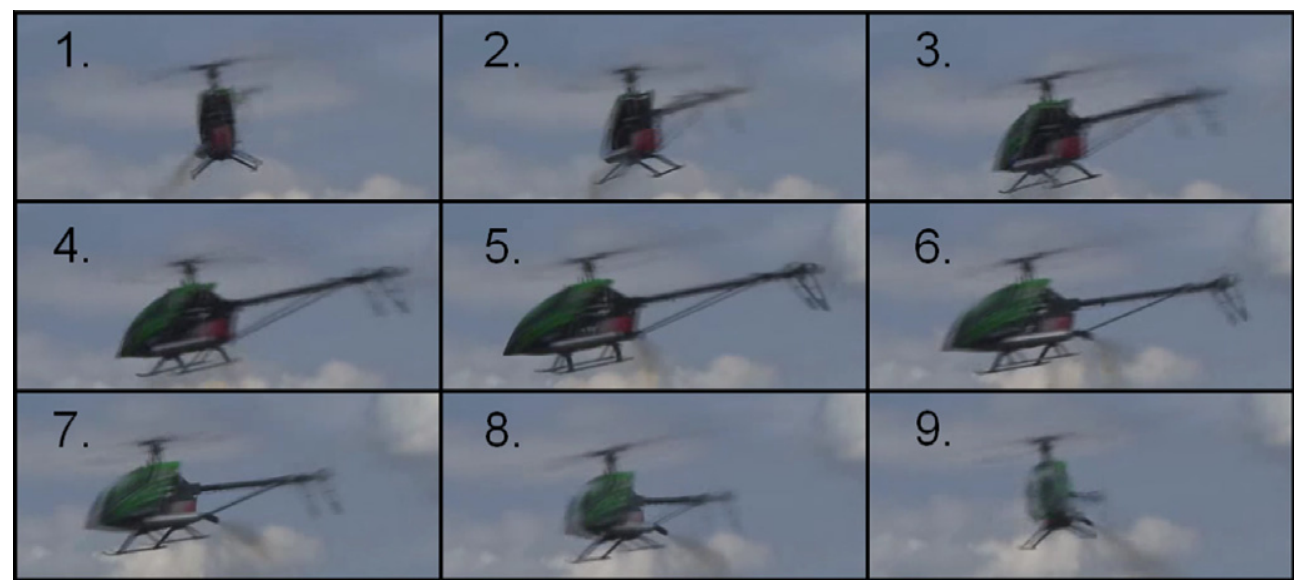

Fig. 9. Frame sequence of anticlockwise pirouette performed in $0.32 \mathrm{~s}$ : the angle is approximately $180^{\circ}$.

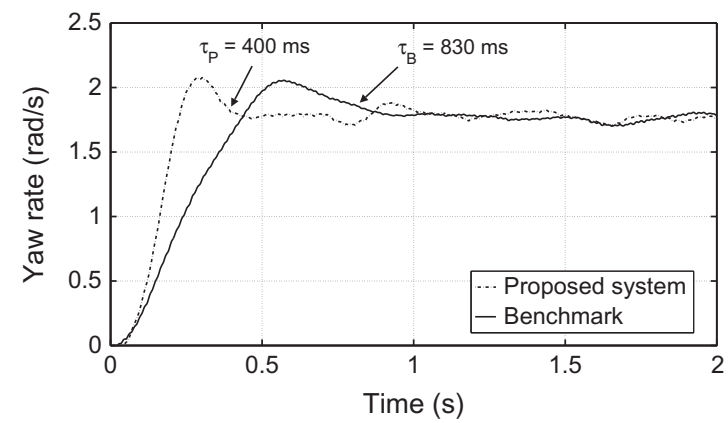

Fig. 10. Response of the proposed system to a $1.75 \mathrm{rad} / \mathrm{s}$ anticlockwise yaw step compared to the benchmark. Arrows represent the setpoint $\pm 5 \%$.

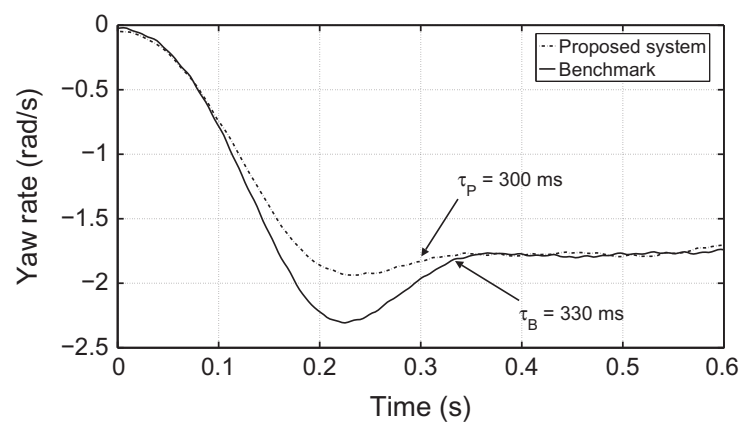

Fig. 11. Response of the proposed system to a $-1.75 \mathrm{rad} / \mathrm{s}$ clockwise yaw step compared to the benchmark. Arrows represent the setpoint $\pm 5 \%$.

formed with the same weather conditions and in absence of significant wind.

Figs. 10 and 11 show the step response of the system when the setpoint was $1.75 \mathrm{rad} / \mathrm{s}$ and $-1.75 \mathrm{rad} / \mathrm{s}$, respectively. Similarly to the simulations results, such low amplitude setpoint guarantees that the performances of the system are characterized independently from the antiwindup algorithm, so Figs. 10 and 11 show the effectiveness of the proposed asymmetric control. As it can be seen

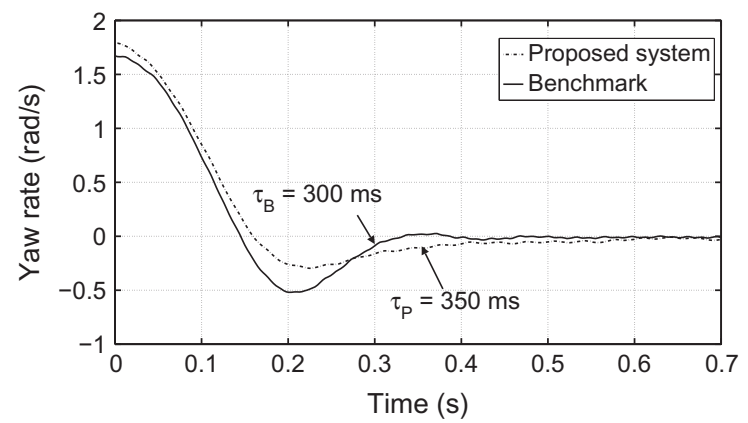

Fig. 12. Response of the proposed system to a stopping step from $1.75 \mathrm{rad} / \mathrm{s}$ anticlockwise pirouette. Arrows represent the setpoint $\pm 5 \%$.

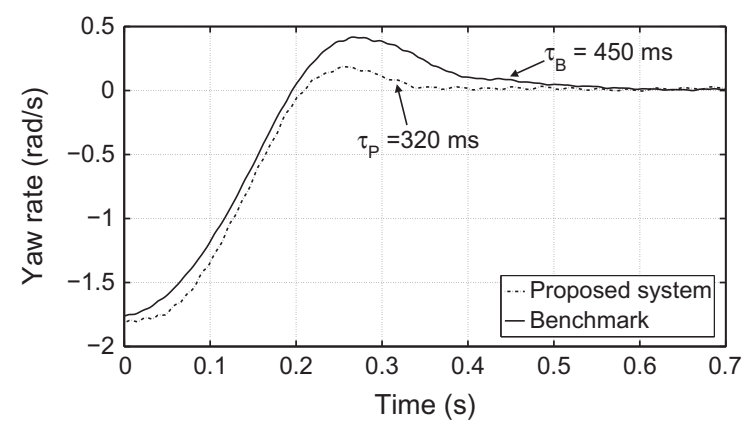

Fig. 13. Response of the proposed system to a stopping step from $-1.75 \mathrm{rad} / \mathrm{s}$ clockwise pirouette. Arrows represent the setpoint $\pm 5 \%$.

also in simulations of Fig. 4, in the clockwise direction the settling time shows only a slight improvement with respect to the benchmark, while in anticlockwise direction the settling time of the proposed system is two times faster.

Figs. 12 and 13 show the settling time of a stopping yaw rate step. Now, the stopping step response from an anticlockwise yaw rate is slightly slower than the benchmark, 


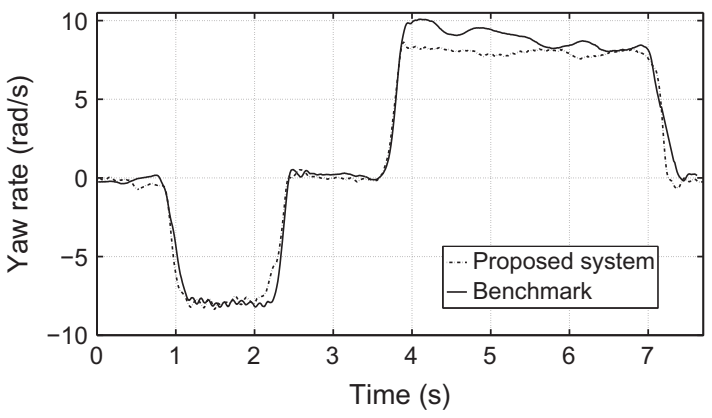

Fig. 14. Qualitative dynamic response of the proposed system compared to the benchmark solution.

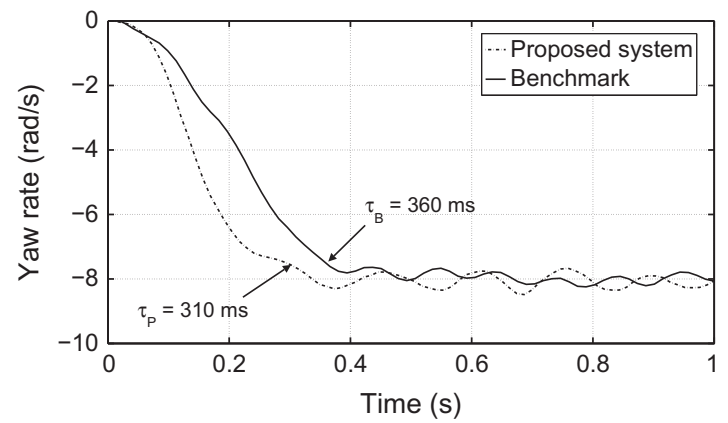

Fig. 15. Response of the proposed system to a $-8 \mathrm{rad} / \mathrm{s}$ clockwise yaw step compared to the benchmark. Arrows represent the setpoint $\pm 5 \%$.

while the response in the other direction is faster by a factor of 1.5 .

Fig. 14 reports the response of the systems to a double step setpoint of $\pm 8 \mathrm{rad} / \mathrm{s}$. Measurement data point out the pirouette constancy, which is a very important issue for $\mathrm{RC}$ pilots. Moreover, the figure shows the settling time at higher rates. By a quantitative analysis, it is possible to obtain information on the effectiveness of the anti-windup algorithm when a high yaw rate is imposed and the actuator is saturated for a great portion of the transient process.

In the clockwise direction, obviously, the actuator is faster and the anti-windup algorithm intervenes for a short time, so that the proposed system is only slightly, and not significantly, faster (Fig. 15).

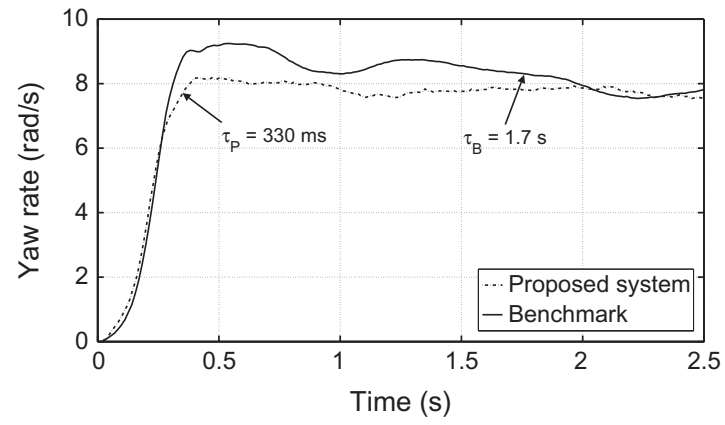

Fig. 16. Response of the proposed system to a $8 \mathrm{rad} / \mathrm{s}$ anticlockwise yaw step compared to the benchmark. Arrows represent the setpoint $\pm 5 \%$.
Table 1

Repeatability measurement obtained in ten cycles of steps.

\begin{tabular}{lll}
\hline $\begin{array}{l}\text { Step type } \\
(\mathrm{rad} / \mathrm{s})\end{array}$ & $\begin{array}{l}\text { Proposed system settling } \\
\text { time }(\mathrm{s})\end{array}$ & $\begin{array}{l}\text { Benchmark settling } \\
\text { time }(\mathrm{s})\end{array}$ \\
\hline $0 \rightarrow 1.75$ & $0.36 \pm 0.02$ & $0.65 \pm 0.11$ \\
$1.75 \rightarrow 0$ & $0.40 \pm 0.03$ & $0.38 \pm 0.04$ \\
$0 \rightarrow-1.75$ & $0.31 \pm 0.01$ & $0.40 \pm 0.02$ \\
$-1.75 \rightarrow 0$ & $0.28 \pm 0.02$ & $0.43 \pm 0.07$ \\
\hline
\end{tabular}

On the contrary, in the anticlockwise direction the proposed system can be more than five times faster than benchmark (Fig. 16). Moreover, the settling times shown in Figs. 10-16 fall all into a very narrow range, assuring a symmetrical helicopter behaviour.

Finally, repeatability measurements were performed with the purpose of accounting for other parameters which may affect measurement results and which are inherent on the "in flight" behaviour (e.g. pilot mistake, wind brief gust, etc.). Repeatability data are derived as type A standard uncertainty evaluated on ten repeated pirouette steps of $\pm 1.75 \mathrm{rad} / \mathrm{s}$ amplitude. The obtained results are summarized in Table 1.

From Table 1, it is apparent that the data corresponding to a clockwise rotation (falling edges) are comparable, while the ones corresponding to anticlockwise rotations (rising edges) point out a faster behaviour for the presented system by a factor of 1.5 .

\section{Conclusions}

The design and characterization of an innovative gyroscopic system for the yaw channel control in UAV aerobatic helicopters have been presented. Differently from existing, state-of-the-art commercial solutions, the proposed system exhibits an almost symmetrical behaviour despite of highly asymmetrical actuation system. Both simulations and measurements performed "in flight" demonstrated a large improvement of overall performances, resulting five times faster in reaching the desired setpoint at higher yaw rates.

\section{Acknowledgment}

We wish to thank ElyQ SpA, Remanzacco (Ud), Italy, and its team pilot for active cooperation and useful hints in describing the performance limitations of existing systems in hard maneuvering.

\section{References}

[1] I.A. Raptis, K.P. Valavanis, Linear and Nonlinear Control of SmallScale Unmanned Helicopters, Springer, 2011.

[2] S.K. Kim, D.M. Tilbury, Mathematical modeling and experimental identification of an unmanned helicopter robot with flybar dynamics, Journal of Robotic Systems 21 (2004) 95-116.

[3] L. Yi-bo, L. Wan-zhu, Sonq-qi, Improved LQG control for small unmanned helicopter based on active model in uncertain environment, IEEE International Conference on Electronics Communications and Control (ICECC) (2011) 289-292.

[4] K. Tang, B. Wang, W. Kang, B. Chen, Minimum time control of helicopter UAVs using computational dynamic optimization, IEEE International Conference on Control and Automation (ICCA) (2011) 848-852. 
[5] F. Leonard, A. Martini, G. Abba, Robust nonlinear controls of modelscale helicopters under lateral and vertical wind gusts, IEEE Transactions on Control Systems Technology 20 (2012) 154-163.

[6] E.N. Johnson, S.K. Kannan, Adaptive trajectory control for autonomous helicopters, Journal of Guidance Control and Dynamics 28 (2005) 524-538.

[7] A.J. Baerveldt, R. Klang, A low-cost and low-weight attitude estimation system for an autonomous helicopter, Proceedings of IEEE Intelligent Engineering Systems (1997) 391-395.

[8] J.S. Jang, D. Liccardo, Small UAV automation using MEMS, Aerospace and Electronic Systems Magazine IEEE 22 (2007) 30-34.

[9] T. Gao, Z. Gong, J. Luo, W. Ding, W. Feng, An attitude determination system for a small unmanned helicopter using low-cost sensors, IEEE Conference on Robotics and Biomimetics (2006) 1203-1208.

[10] J. Wang, Y. Liu, W. Fan, Design and calibration for a smart inertial measurement unit for autonomous helicopters using MEMS sensors, IEEE Conference on Mechatronics and Automation (2006) 956-961.

[11] G. Cai, K. Peng, B.M. Chen, T.H. Lee, Design and assembling of a UAV helicopter system, in: Proceedings of the 5th International Conference on Control and Automation, 2005, pp. 697-702.

[12] G. Cai, B.M. Chen, K. Peng, M. Dong, T.H. Lee, Modeling and control of the yaw channel of a UAV helicopter, IEEE Transactions on Industrial Electronics 55 (2008) 3426-3434.

[13] V. Gavrilets, A. Shterenberg, M. Dahleh, E. Feron, Avionics system for a small unmanned helicopter performing aggressive maneuvers, in: Proceedings of the 19th Digital Avionics Systems Conferences, vol. 1, 2000, pp. 1-7 (1E2).
[14] V. Gavrilets, A. Shterenberg, I. Martinos, K. Sprague, M.A. Dahleh, E. Feron, Avionics systems for aggressive maneuvers, IEEE AESS Systems Magazine (2001) 38-43.

[15] Isidori A, L. Marconi, A. Serrani, Robust nonlinear motion control of a helicopter, IEEE Transactions on Automatic Control 48 (2003) 413426.

[16] G. Heredia, A. Ollero, M. Bejar, R. Mahtani, Sensor and actuator fault detection in small autonomous helicopters, Mechatronics 18 (2008) 90-99.

[17] http://www.rcheliwiki.com/Category:Maneuvers

[18] G. Wang, J. Zhu, C. Yang, System identification for helicopter yaw dynamic modelling, IEEE International Conference on Computer Research and Development (ICCRD) (2011) 54-57.

[19] X. Wang, X. Zhao, M. Tan, Modeling, identification and robust control of yaw dynamics of small-scale unmanned helicopters, IEEE Fifth International Conference on Natural Computation (2009) 273-276.

[20] A. Affanni, Gyroscopic system for yaw channel control in aerobatic UAV helicopters, in: Proceedings of IEEE Sesnors and Applications Symposium SAS 2012, 2012, pp. 217-221.

[21] Analog Devices ADXRS620 Data Sheet Rev B, 09/2010. <http:// www.analog.com/en/mems-sensors/mems-inertial-sensors/ adxrs620/products/product.html>.

[22] A. Scottedward Hodel, C.E. Hall, Variable-structure PID control to prevent integrator windup, IEEE Transactions on Industrial Electronics 48 (2001) 442-451.

[23] A. Visioli, Modified anti-windup scheme for PID controllers, IEEE Control Theory and Applications 150 (2003) 49-54. 